\title{
Immune checkpoint inhibitors: recent progress and potential biomarkers
}

\author{
Pramod Darvin', Salman M. Toor', Varun Sasidharan Nair ${ }^{1}$ and Eyad Elkord (1] ${ }^{1,2}$
}

\begin{abstract}
Cancer growth and progression are associated with immune suppression. Cancer cells have the ability to activate different immune checkpoint pathways that harbor immunosuppressive functions. Monoclonal antibodies that target immune checkpoints provided an immense breakthrough in cancer therapeutics. Among the immune checkpoint inhibitors, PD-1/PD-L1 and CTLA-4 inhibitors showed promising therapeutic outcomes, and some have been approved for certain cancer treatments, while others are under clinical trials. Recent reports have shown that patients with various malignancies benefit from immune checkpoint inhibitor treatment. However, mainstream initiation of immune checkpoint therapy to treat cancers is obstructed by the low response rate and immune-related adverse events in some cancer patients. This has given rise to the need for developing sets of biomarkers that predict the response to immune checkpoint blockade and immune-related adverse events. In this review, we discuss different predictive biomarkers for anti-PD-1/PD-L1 and anti-CTLA-4 inhibitors, including immune cells, PD-L1 overexpression, neoantigens, and genetic and epigenetic signatures. Potential approaches for further developing highly reliable predictive biomarkers should facilitate patient selection for and decision-making related to immune checkpoint inhibitor-based therapies.
\end{abstract}

\section{Introduction}

The development of immune checkpoint inhibitors (ICIs) is a revolutionary milestone in the field of immunooncology. Tumor cells evade immunosurveillance and progress through different mechanisms, including activation of immune checkpoint pathways that suppress antitumor immune responses. ICIs reinvigorate antitumor immune responses by interrupting co-inhibitory signaling pathways and promote immune-mediated elimination of tumor cells.

Ipilimumab, which targets cytotoxic T-lymphocyte antigen-4 (CTLA-4), was the first approved immune checkpoint inhibitor for treating patients with advanced melanoma ${ }^{1-3}$. This antibody prevents $\mathrm{T}$-cell inhibition

Correspondence: Eyad Elkord(eelkord@hbku.edu.qa) (eyad.elkord@manchester. ac.uk)

${ }^{1}$ Cancer Research Center, Qatar Biomedical Research Institute, College of

Science and Engineering, Hamad Bin Khalifa University, Qatar Foundation, Doha, Qatar

${ }^{2}$ Institute of Cancer Sciences, University of Manchester, Manchester, UK and promotes the activation and proliferation of effector $\mathrm{T}$ cells. Following the approval of ipilimumab, other antibodies that target immune checkpoints were examined. Currently, hundreds of phase I and II clinical trials and phase III/IV clinical trials are being carried out across the globe to evaluate the efficacy of multiple ICIs as monotherapy or in combination (details of phase III/IV trials are given in Table 1).

Pembrolizumab and nivolumab, ICIs that target programmed death-1 (PD-1), showed promising results in melanoma and non-small cell lung carcinoma (NSCLC) patients, with an objective response rate (ORR) of $40-45 \%^{4-6}$. Additionally, urothelial bladder cancer patients treated with PD-1/PD-L1 inhibitors showed an increase in overall response rate, between 13 and $24 \%^{7}$. In triple-negative breast cancer (TNBC) patients, the response to PD-1 inhibitors was relatively moderate $(19 \%)^{8}$. In contrast, in relapsed or refractory Hodgkin's lymphoma, nivolumab showed an objective response rate of $87 \%$ with $17 \%$ complete response ${ }^{9}$. Pembrolizumab and 
Table 1 Immune checkpoint inhibitors in phase III and IV clinical trials

\begin{tabular}{|c|c|c|c|}
\hline SI No & Drug & Cancer type & Clinical trial ID \\
\hline 1 & Pembrolizumab (Anti-PD-1) & NSCLC & $\begin{array}{l}\text { NCT03134456, NCT02220894, NCT02142738, NCT02864394, } \\
\text { NCT03302234, NCT01905657, NCT02504372, NCT02775435, } \\
\text { NCT02578680 }\end{array}$ \\
\hline 2 & & Small cell lung cancer & NCT03066778 \\
\hline 3 & & Head and neck squamous cell carcinoma & NCT02252042, NCT03040999, NCT02358031 \\
\hline 4 & & Renal cell carcinoma & NCT03142334, NCT02853331 \\
\hline 5 & & Gastric adenocarcinoma & NCT02370498 \\
\hline 6 & & Nasopharyngeal neoplasms & NCT02611960 \\
\hline 7 & & Urothelial carcinoma & $\begin{array}{l}\text { NCT02853305, NCT03244384, NCT02256436, NCT03374488, } \\
\text { NCT03361865 }\end{array}$ \\
\hline 8 & & Colorectal cancer & NCT02563002 \\
\hline 9 & & Pleural mesothelioma & NCT02991482 \\
\hline 10 & & TNBC & NCT02819518, NCT03036488, NCT02555657 \\
\hline 11 & & Esophageal neoplasms & NCT03189719, NCT02564263 \\
\hline 12 & & Multiple myeloma & NCT02579863, NCT02576977 \\
\hline 13 & & Gastric and gastroesophageal junction cancer & NCT03019588, NCT03221426 \\
\hline 14 & & Gastric adenocarcinoma & NCT02494583 \\
\hline 15 & & Melanoma & NCT02362594, NCT01866319 \\
\hline 16 & & Hodgkin lymphoma & NCT02684292 \\
\hline 17 & & Hepatocellular carcinoma & NCT02702401, NCT03062358 \\
\hline 18 & & Lung cancer & NCT03322540 \\
\hline 19 & & Head and neck cancer & NCT03358472 \\
\hline 20 & Nivolumab (Anti-PD-1) & NSCLC & NCT02041533, NCT01642004, NCT01673867 \\
\hline 21 & & Mesothelioma & NCT03063450 \\
\hline 22 & & Non-Hodgkin lymphoma & NCT03366272 \\
\hline 23 & & Metastatic clear cell renal carcinoma & NCT01668784 \\
\hline 24 & & Head and neck cancer & NCT02741570, NCT03342352 \\
\hline 25 & & Lung cancer & NCT03348904 \\
\hline 26 & & Melanoma & NCT03068455, NCT01844505 \\
\hline 27 & Ipilimumab (Anti-CTLA-4) & NSCLC & NCT03469960, NCT03351361, NCT02785952, NCT03302234 \\
\hline 28 & & Squamous cell lung carcinoma & NCT02785952 \\
\hline 29 & & Mesothelioma & NCT02899299 \\
\hline 30 & & $\begin{array}{l}\text { Gastric cancer } \\
\text { Gastroesophageal junction cancer }\end{array}$ & NCT02872116 \\
\hline 31 & & Metastatic melanoma & $\begin{array}{l}\text { NCT03445533, NCT00636168, NCT01274338, NCT02339571, } \\
\text { NCT02506153, NCT02224781, NCT00094653 }\end{array}$ \\
\hline 32 & & Metastatic non-cutaneous melanoma & NCT02506153 \\
\hline 33 & Avelumab (Anti-PD-L1) & NSCLC & NCT02576574, NCT02395172 \\
\hline 35 & & Urothelial cancer & NCT02603432 \\
\hline 35 & & Diffuse Large B-cell lymphoma & NCT02951156 \\
\hline
\end{tabular}


Table 1 continued

\begin{tabular}{|c|c|c|c|}
\hline SI No & Drug & Cancer type & Clinical trial ID \\
\hline 36 & & Renal cell cancer & NCT02684006 \\
\hline 37 & & Gastric and gastroesophageal junction cancer & NCT02625623, NCT02625610 \\
\hline 40 & Atezolizumab (Anti-PD-L1) & $\begin{array}{l}\text { Ovarian cancer, fallopian tube cancer } \\
\text { Peritoneal neoplasms }\end{array}$ & NCT03038100, NCT02839707, NCT02891824 \\
\hline 41 & & NSCLC & $\begin{array}{l}\text { NCT02813785, NCT02008227, NCT02367781, NCT02366143, } \\
\text { NCT02409342, NCT02486718, NCT02367794, NCT03191786, } \\
\text { NCT02409355, NCT02657434, NCT03456063 }\end{array}$ \\
\hline 42 & & Extensive stage small cell lung cancer & NCT02763579 \\
\hline 43 & & TNBC & NCT03197935, NCT02425891, NCT03125902, NCT03281954 \\
\hline 44 & & Renal cell carcinoma & NCT02420821, NCT03024996 \\
\hline 45 & & Bladder cancer & NCT02302807 \\
\hline 46 & & Squamous cell carcinoma of the head and neck & NCT03452137 \\
\hline 47 & & Urothelial carcinoma & NCT02807636 \\
\hline 48 & & Transitional cell carcinoma & NCT02450331 \\
\hline 49 & & Prostatic neoplasms & NCT03016312 \\
\hline 50 & Durvalumab (Anti-PD-L1) & NSCLC & $\begin{array}{l}\text { NCT02352948, NCT03003962, NCT02453282, NCT02273375, } \\
\text { NCT02542293, NCT03164616, NCT02125461, }\end{array}$ \\
\hline 51 & & Squamous cell lung carcinoma & NCT02154490, NCT02551159 \\
\hline 52 & & $\begin{array}{l}\text { Recurrent or metastatic PD-L1 positive or } \\
\text { negative SCCHN }\end{array}$ & NCT02369874 \\
\hline 53 & & Recurrent squamous cell lung caner & NCT02766335, NCT02154490 \\
\hline 54 & & Urothelial cancer & NCT02516241 \\
\hline 55 & & Advanced solid malignancies & NCT03084471 \\
\hline 56 & & $\begin{array}{l}\text { SCCHN, hypo pharyngeal squamous cell } \\
\text { carcinoma, laryngeal squamous cell carcinoma }\end{array}$ & NCT02551159, NCT03258554 \\
\hline 57 & REGN2810 (Anti-PD-1) & NSCLC & NCT03409614, NCT03088540 \\
\hline 58 & BMS-936558 (Anti-PD-1) & Unresectable or metastatic melanoma & NCT01721746, NCT01721772 \\
\hline 59 & SHR1210 (Anti-PD-1) & NSCLC & NCT03134872 \\
\hline 60 & & Nasopharyngeal neoplasms & NCT03427827 \\
\hline 61 & KN035 (Anti-PD-L1) & Biliary tract neoplasms & NCT03478488 \\
\hline 62 & IBI308 (Anti-PD-1) & Squamous cell lung carcinoma & NCT03150875 \\
\hline 63 & PDR001 (Anti-PD-1) & Melanoma & NCT02967692 \\
\hline 64 & Anti-PD-1 & Metastatic melanoma & NCT02821013 \\
\hline 65 & BGB-A317 (Anti-PD-1) & $\mathrm{NSCLC}$ & NCT03358875 \\
\hline 66 & & Esophageal squamous cell carcinoma & NCT03430843 \\
\hline 67 & & Hepatocellular carcinoma & NCT03412773 \\
\hline 68 & BCD-100 (Anti-PD-1) & NSCLC & NCT03288870 \\
\hline 70 & JS001 (Anti-PD-1) & Metastatic melanoma & NCT03430297 \\
\hline
\end{tabular}


nivolumab are currently under phase IV clinical trials for treating various malignancies (Table 1).

Despite the success of anti-CTLA-4 and anti-PD-1/PDL1 therapies, only a fraction of patients benefit from ICIs. Antitumor immunity, regulated through complex factors in the tumor microenvironment (TME), could create variable immune responses. The TME is segregated into three major types based on the infiltration of immune cells: immune desert, immune excluded and immune inflamed ${ }^{10}$. These phenotypes have their own mechanisms for preventing immune responses from eradicating tumor cells ${ }^{10}$. Immune deserts are characterized by the absence of $\mathrm{T}$ cells in the TME and the lack of suitable $\mathrm{T}$ cell priming or activation. The immune excluded phenotype exhibits the presence of multiple chemokines, vascular factors or mediators and stromal-based inhibition; however, accumulated $\mathrm{T}$ cells are unable to infiltrate the TME. Immune inflamed tumors demonstrate infiltration of multiple immune cell subtypes ${ }^{10}$.

Accumulating evidence suggests that only a fraction of cancer patients benefit from checkpoint inhibitors, and severe immune-related adverse events (irAEs) are seen in some patients undergoing ICI therapy ${ }^{11}$. irAEs are due to the inhibition of immune checkpoints that reinforce the normal physiological barriers against autoimmunity, leading to various local and systemic autoimmune responses. Therefore, the development of predictive biomarkers is critical for differentiating responders and nonresponders to avoid any adverse effects. Ongoing clinical studies are aiming to develop predictive biomarkers for better treatment outcomes and less irAEs.

Predictive biomarkers could determine the outcome of therapy in a patient before the initiation of a proposed therapy. These biomarkers should indicate whether a patient would benefit from a particular checkpoint monotherapy or if there is a need for combination therapy. In this review, we discuss biomarkers that predict the response to various ICI therapies in cancer.

\section{Immune cells}

Immune inflamed tumors have a high degree of response to immunotherapy. Reports suggest that immune inflamed tissues are more sensitive because ICIs can activate immune reactions and inhibit immune evasion/suppression. Studies confirmed that the response to ICI therapy is related to tumor-infiltrating lymphocytes (TILs) and other immune cells in the TME ${ }^{12}$.

Analyses of peripheral blood is a noninvasive method with good potential to predict treatment outcomes after immune therapies. Reports have shown that in various malignancies, increased tumor-infiltrating immune cells and peripheral blood absolute lymphocyte count (ALC) can be utilized as predictive biomarkers ${ }^{13-15}$. The role of ALC as a predictive biomarker has been validated in metastatic melanoma patients treated with ipilimumab. Patients with 1.35-fold higher ALC values from baseline in the first 2 weeks of treatment had significantly higher overall survival ${ }^{16}$. In ipilimumab-treated patients, overall progression-free survival was associated with a low serum lactate dehydrogenase value ( $\mathrm{LDH} \leq 1.2$-fold), a low absolute monocyte count (AMC $<650$ cells $/ \mu \mathrm{L}$ ), a low myeloid-derived suppressor cell count (MDSCs $<5.1 \%$ ), a high absolute eosinophil count (eosinophils $\geq 50$ cells $/ \mu \mathrm{L}$ ), a relative lymphocyte count $<10.5 \%$ and baseline $\mathrm{CD} 4{ }^{+} \mathrm{CD} 25^{+} \mathrm{FOXP}^{+}$Tregs $\geq 1.5 \%$ in the peripheral blood $^{15,17}$. Multiple studies validating the applicability of LDH as a predictive biomarker showed that patients with elevated levels of LDH also responded to $\mathrm{ICIs}^{18}$. Studies have reported that $\mathrm{LDH}$ can be used as a potential predictive biomarker for overall survival but not as a prognostic biomarker ${ }^{15,16,19}$. CyTOF-based immune profiling of peripheral blood samples collected from anti-CTLA-4 and anti-PD-1-treated melanoma patients showed a distinct set of biomarkers in response to therapy ${ }^{20}$. This study suggested that the abundance of $\mathrm{CD}^{+}$and $\mathrm{CD} 8^{+}$ memory $\mathrm{T}$ cells was a predictive biomarker for antiCTLA-4 therapy and the abundance of $\mathrm{CD}^{+} 9^{+}$and MIP $1 \beta^{+}$NK cells was a predictive biomarker for anti-PD1 therapy $^{20}$. CyTOF analyses of anti-PD-1-treated melanoma patients showed an involvement of CD $14^{+}$CD $16^{-}$HLA-DR $^{\text {hi }}$ cells in therapy response and progression-free survival (PFS ${ }^{21}$. An increase in circulating $\mathrm{CD}^{+}, \mathrm{CD}^{+} \mathrm{T}$ cells and ALC, 2 to 8 weeks after treatment initiation with ipilimumab, was reported in melanoma patients with better clinical outcomes ${ }^{22}$. Apart from the circulating $\mathrm{CD} 8^{+} \mathrm{T}$ cells, $\mathrm{CD} 8^{+}$effector memory type- $1 \mathrm{~T}$ cells were also reported as predictive biomarkers for ipilimumab-treated stage IV melanoma patients ${ }^{23,24}$.

The presence of TILs in various malignancies can be used as potent predictive biomarkers for response to $\mathrm{ICIs}^{13,14}$. Tumors with increased TILs are a major hallmark of the immune inflamed phenotype, and they exhibit improved immune-mediated elimination of tumor cells. In ipilimumab-treated melanoma patients, TILs were significantly increased from baseline in a therapyresponsive group, confirming their significance in response to $\mathrm{ICIs}^{25}$. To explain the role of immune cells in the treatment response, a study was carried out using 52 lymph nodes and 34 cutaneous/subcutaneous metastatic surgical samples collected from 30 metastatic melanoma patients receiving ipilimumab ${ }^{26}$. In this study, Balatoni et al. ${ }^{26}$ examined 11 immune cell subsets in the TME and their post-therapy responses. Interestingly, 7 out of 11 immune subsets positively correlated with an increase in the overall survival rate. These subsets included $\mathrm{CD} 4^{+}$ $\mathrm{T}$ cells, $\mathrm{CD}^{+} \mathrm{T}$ cells, $\mathrm{CD} 20^{+} \mathrm{B}$ cells, cells expressing $\mathrm{CD}_{134^{+}}$and $\mathrm{CD} 137^{+}$activation markers, $\mathrm{FOXP}^{+}{ }^{+} \mathrm{T}$ cells and NKp $46^{+}$cells. Notably, subcutaneous and cutaneous 
metastatic tissues, compared to lymph nodes, showed distinct immune cell infiltration. In subcutaneous and cutaneous samples, the presence of $\mathrm{CD} 16^{+}$and $\mathrm{CD} 68^{+}$ cells positively correlated with therapy response as well as prolonged survival. In contrast, in the lymph nodes, $\mathrm{CD}_{45 \mathrm{RO}^{+}}, \mathrm{PD}-1^{+}, \mathrm{CD} 16^{+}$, and $\mathrm{CD} 68^{+}$cells correlated only with increased survival ${ }^{26}$. In addition to the abundance of $\mathrm{FOXP}^{+}$Tregs, the ratio of effector $\mathrm{T}$ cells (Teffs) to Tregs is reported to be a more specific predictive biomarker for anti-CTLA-4 immune therapies ${ }^{27,28}$. Immune profiling of TILs using multiparametric flow cytometry in metastatic melanoma patients showed that PD- $1{ }^{\text {hi }}$ CTLA- $4{ }^{\text {hi }}$ in $\mathrm{CD} 8^{+}$T cells predict the response to anti-PD-1 therapy ${ }^{29}$. This study is supported through the identification of both transcriptionally and functionally distinct $\mathrm{CD}^{+}{ }^{+} \mathrm{PD}-1^{+}$T-cell subpopulations in NSCLC patients, showing predictive potential for anti-PD-1 therapy $^{30}$. Additionally, intratumoral and peripheral $\mathrm{CD}^{+}{ }^{+} \mathrm{FOXP3}{ }^{-}{ }^{\mathrm{PD}}-1^{\text {hi }}$ nonconventional Tregs in NSCLC as well as melanoma patients were reported as prognostic biomarkers for anti-PD-1 and anti-CTLA-4 therapies ${ }^{31}$. Anti-CTLA-4 therapy induced an immune inflamed phenotype via expansion of intratumoral and systemic CD $4^{+}$FOXP3 ${ }^{-}$PD $-1^{\text {hi }}$ Tregs that were reduced with antiPD-1 therapy and improved the overall antitumor response ${ }^{31}$. Moreover, $\mathrm{PD}-\mathrm{L} 1^{+} \mathrm{CD} 4^{+} \mathrm{CD} 25^{+}$Tregs predict responses to PD-1/PD-L1 blockade in NSCLC patients ${ }^{32}$. Figure 1 shows an overview of how the presence of various immune cell subsets in the TME may contribute to the differential responses to ICIs in responders and nonresponders.

Pembrolizumab in advanced melanoma patients showed that pre-existing $\mathrm{CD}^{+} \mathrm{T}$ cells in the TME are required for better tumor regression ${ }^{33}$. The presence of an immune excluded phenotype with an abundance of immune cells at invasive margins or stroma is also associated with clinical benefits. The spatiotemporal dynamics of $\mathrm{CD} 8^{+} \mathrm{T}$ cells are also an important factor for better treatment outcomes. Analysis of pretreatment samples collected from patients undergoing PD-1/PD-L1 therapy showed a relatively higher abundance of $\mathrm{CD}^{+} \mathrm{T}$ cells at the invasive margins in therapy responders. These pretreatment samples show an immune excluded phenotype through increased accumulation of $\mathrm{T}$ cells on the invasive margin without effective infiltration. Moreover, serially sampled tumors during therapy showed an increase in $\mathrm{CD} 8^{+} \mathrm{T}$ cells at the invasive margin and then in parenchyma in the response group ${ }^{33}$. This increase in $\mathrm{CD} 8^{+} \mathrm{T}$ cells may be due to the negative regulation of PD-1/PD-L1 by ICIs, which resulted in either the infiltration of immune cells or the enhanced proliferation of $\mathrm{CD}^{+} \mathrm{T}$ cells ${ }^{33}$. Additionally, it has been reported that in lung cancer patients, $\mathrm{CD} 3^{+}, \mathrm{CD} 4^{+}$and $\mathrm{CD}^{+} \mathrm{T}$-cell infiltration to deep tissues significantly correlated with longer overall survival ${ }^{34}$. Metastatic breast cancer patients treated with atezolizumab showed an increased ORR related to stromal TILs ${ }^{35}$. The predictive potentials of stromal TILs were confirmed in the KEYNOTE-086 study; significantly higher levels of stromal TILs were associated with the anti-PD-1 therapy response in metastatic triple-negative breast cancer patients ${ }^{36}$.

CTLA-4 blockade activates $T$ cells to target malignant cells. CTLA- 4 is constitutively expressed in T cells and attenuates immune responses when bound to CD80 or CD86 on the surface of antigen-presenting cells (APCs) (Fig. 2). Analyses of pre- and post-treated surgical tissues and peripheral blood showed that the inducible costimulator (ICOS) pathway is activated upon anti-CTLA-4 therapy ${ }^{37}$. This overexpression of ICOS (CD28/CTLA-4 Ig superfamily) resulted in an increase in $\mathrm{ICOS}^{+} \mathrm{T}$ cells in both tumor and blood samples ${ }^{37}$. In tremelimumabtreated breast cancer patients, increased $\mathrm{CD}_{4}^{+} \mathrm{ICOS}^{+}$and $\mathrm{CD}^{+}{ }^{+} \mathrm{ICOS}^{+} \mathrm{T}$ cells were observed in peripheral blood ${ }^{37}$. The ratio of FOXP3 ${ }^{+}$Treg cells to $\mathrm{ICOS}^{+} \mathrm{T}$ cells was also increased in therapy-responsive patients ${ }^{37}$. Moreover, in patients exhibiting clinical benefits, there was an increase in the frequency of $\mathrm{CD}^{+}{ }^{+} \mathrm{ICOS}^{+}$Teff cells. These cells express T-bet and produce IFN- $\gamma$, strengthening immune responses in anti-CTLA-4 therapy ${ }^{38-40}$.

\section{PD-L1 overexpression}

Interactions between PD-1 and its ligands, B7-H1/PD$\mathrm{L} 1$ and B7-DC/PD-L2, lead to T-cell inactivation to maintain immune homeostasis and prevent autoimmunity. PD-1/PD-L1 pathway activation is related to the immune inflamed phenotype ${ }^{41}$. IC ligands are commonly found on tumor cells, and these interactions work in tandem with elevated tumor infiltration of immunosuppressive cells to support tumor escape from active Tcell responses ${ }^{42}$. Therefore, blocking the PD-1/PD-L1 inhibitory pathway can activate $\mathrm{T}$ cells in the TME, releasing inflammatory cytokines and cytotoxic granules to eliminate tumor cells (Fig. 2).

The direct approach to check responsiveness to PD-1/ PD-L1 therapy in patients is to detect the expression levels of PD-L1 in tumor tissues. Teng et al. ${ }^{43}$ proposed four different classifications of TME based on the presence of TILs and PD-L1 expression. They classified PDL1-positive tumors with TILs as a type I tumor microenvironment and proposed it to be most likely to respond to immune checkpoint blockade.

IHC analyses performed on patients with metastatic melanoma, NSCLC, colon cancer, renal cell carcinoma and prostate cancer who underwent PD-1/PD-L1 targeting therapy suggested PD-L1 overexpression as a potential biomarker. An open-label Phase II clinical trial of pembrolizumab in NSCLC reported that progression-free survival and overall survival were higher in patients with PD-L1 expression in at least $50 \%$ of tumor cells ${ }^{44}$. 

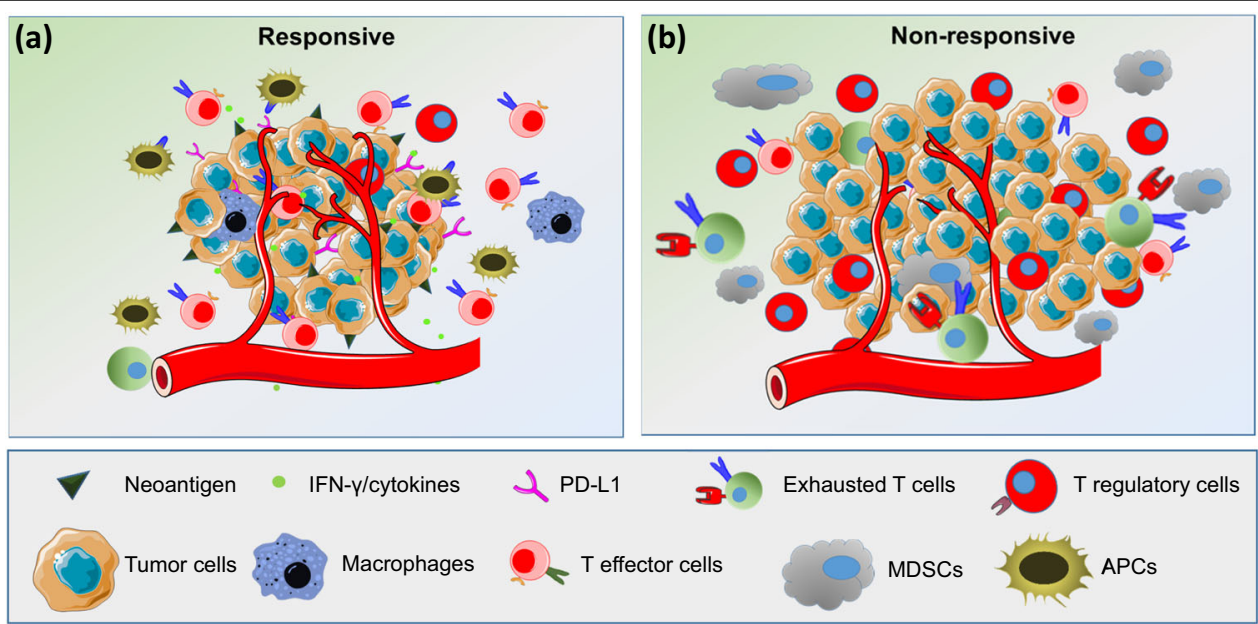

Fig. 1 Overview of predictive biomarkers for response to ICIs. The response to immune checkpoint inhibitors varies depending on the TME. In the responders, tumors have a high neoantigen load, high levels of TILs, especially effector cells, a high Teff to Treg ratio, low MDSC levels and increased secretion of IFN- $\gamma$ and other cytokines (a). In nonresponders, the TME contains high levels of immunosuppressive cells, such as Tregs and MDSCs, and very low levels of NK cells and activated lymphocytes (b)

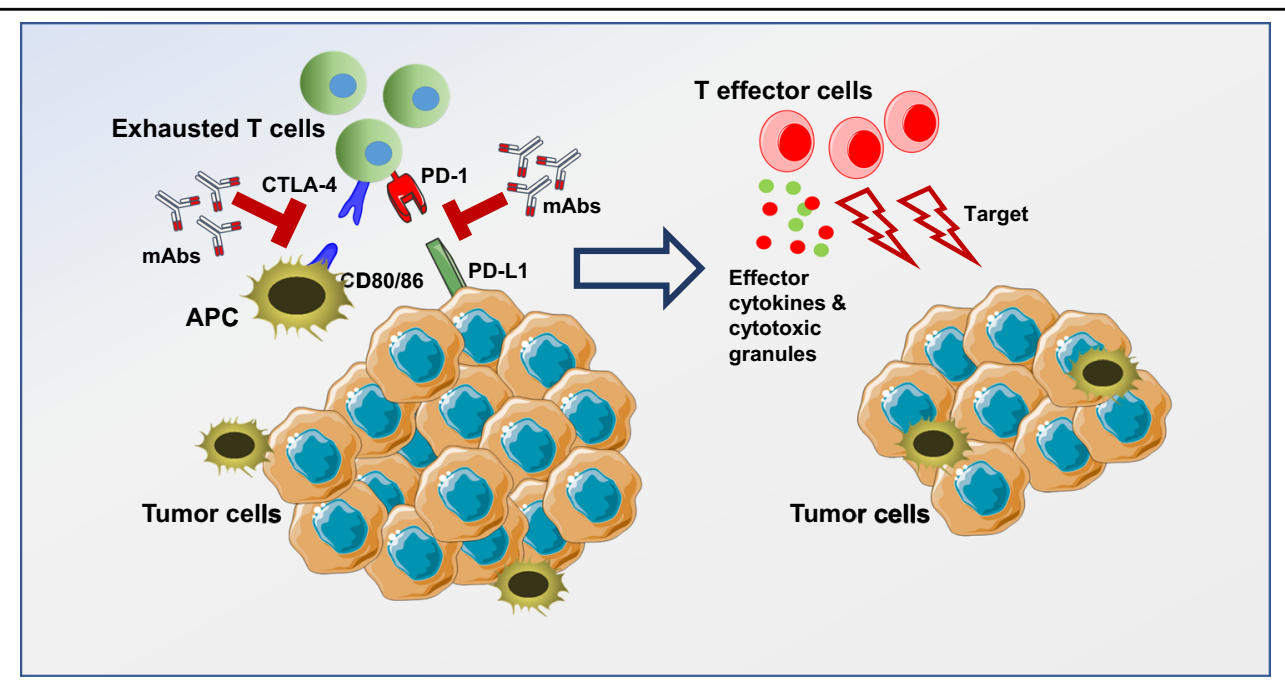

Fig. 2 Immune checkpoint blockade for T-cell activation. Immune checkpoints, including PD-1 and CTLA-4, expressed on activated T cells lead to inhibition of T-cell activation upon binding to their ligands on tumor cells/antigen-presenting cells. These interactions can be blocked using monoclonal antibodies, leading to the activation of T cells targeting tumor cells through the release of effector cytokines and cytotoxic granules.

Notably, elevated levels of PD-L1 expression in the TME do not correlate with worse differentiation and poor prognosis. High PD-L1 expression is often accompanied by IFN- $\gamma$-secreting TILs in some cancers ${ }^{45}$. However, Aguiar et al. suggested that PD-L1 overexpression may not be a robust biomarker for the response to ICIs in all cancers, as PD-L1-negative tumors can also respond to $\mathrm{mAbs}$ targeting PD-1/PD-L1 interactions. Therefore, to date, $\mathrm{PD}-\mathrm{L} 1$ overexpression as a prerequisite for initiation of PD-1/PD-L1 checkpoint blockade is not established as a potent biomarker for determining responsiveness to anti-PD-1/PD-L1 based immunotherapy.
Investigating $\mathrm{PD}-\mathrm{L} 1$ expression has some limitations that need to be considered. PD-L1 expression is known to be both spatial and temporal, and it is also expressed on other immune cells, including antigen-presenting cells. One plausible approach to counter these limitations is to perform PD-L1 expression analyses on circulating tumor cells (CTCs) in peripheral blood samples from cancer patients. Interestingly, $\mathrm{PD}-\mathrm{L} 1^{+} \mathrm{CTCs}$ were found to be higher than PD-L1 ${ }^{+}$cells in the TME of NSCLC patients ( $83 \%$ vs. $41 \%$ ), and no correlation was observed between tissue and CTC PD-L1 expression ${ }^{46}$. Therefore, further investigations are warranted to 
establish PD-L1 expression on CTCs as a predictive biomarker.

\section{Neoantigens}

Acquired mutations during cancer progression have promise in detecting efficiency of and resistance to therapy. Mutations in the protein-coding regions of DNA generate truncated proteins termed "neoantigens." Neoantigens result in a higher degree of foreignness to cells, which helps immune cells readily target and eliminate tumor cells. Various neoantigens that confer therapy efficacy could be potential biomarkers for predicting the clinical activity of ICIs. A retrospective study on stage I/II and stage III/IV lung cancer samples showed that high neoantigen burden is associated with the longest overall survival $(P=0.025)^{47}$. Moreover, intratumoral heterogeneity analyses showed that high neoantigen-expressing clones were homogenous with the highest differential expression of PD-L1 and IL- $6{ }^{47}$. Additionally, CD8 $\alpha$ and $\beta$, STAT1, TAP-1 and 2, CXCL-10, CXCL-9, granzyme $-\mathrm{B},-\mathrm{H}$, and $-\mathrm{A}$, and $\mathrm{IFN}-\gamma$ were upregulated in the high neoantigen-expressing clones ${ }^{47}$. Overexpression of IFN- $\gamma$, IDO, and Th1-associated markers was reported in ipilimumab-treated patients with favorable clinical outcomes. Resistance to CTLA-4 therapy was observed with a loss in IFN- $\gamma$ signaling in $\mathrm{CD}^{+} \mathrm{T}$ cells. These findings confirm that the immune-mediated elimination of tumor cells could be proportional to the neoantigen load. Neoantigens exhibiting high-affinity binding with MHC and TCR are highly eliminated neoantigens ${ }^{48}$. Moreover, acquired resistance to ICI can also be predicted through neoantigen landscapes ${ }^{48}$. Screening of these neoantigens has the potential to predict clinical activity as well as therapeutic resistance.

Tumor tissues from melanoma patients treated with ipilimumab or tremelimumab were used to study the role of somatic mutations as predictive biomarkers for clinical response. Whole genome somatic neoepitope analyses and patient-specific HLA typing were performed in tumors and whole blood samples from 64 patients. It was reported that the neoantigen landscape, as defined through IHC analyses, has a strong association with the treatment response to CTLA-4 blockade ${ }^{49}$. This study strengthens high-throughput IHC analyses using biopsy specimens to clinically validate therapeutic outcomes.

Recent studies revealed that the evolution of the neoantigen profile in NSCLC patients is associated with the response to ICIs. Acquired resistance to immunotherapy was observed in a cohort of 42 patients with NSCLC who were treated with a PD-1 inhibitor alone or in combination with a CTLA- 4 inhibitor ${ }^{48}$. The whole genome of paired tissues collected before and after therapy was analyzed for the neoantigen landscape related to therapy resistance. This study reported that loss-of-function mutations coding for neoantigens either by the elimination of tumor clones or by chromosomal truncated gene alteration can result in therapy resistance ${ }^{48}$. Additionally, tumor cells alter the expression of immune suppressive proteins and multiple transcription factors involved in immune functions to acquire resistance against $\mathrm{ICIs}^{50}$. Whole-genome analyses performed on tissues obtained from baseline and relapsed tumors of metastatic melanoma patients undergoing pembrolizumab treatment revealed that acquired resistance to ICIs are associated with loss-of-function mutations ${ }^{51}$. Truncated mutations in IFN-receptor-associated JAK1 or JAK2 that cause the loss of IFN- $\gamma$ function and mutations in the B2M gene, resulting in the loss of MHC-I expression and antigen presentation, are also reported in acquired ICI therapy-resistant samples ${ }^{51}$.

\section{Genetic signatures}

In a retrospective study conducted with a cohort of breast cancer patients with 1- to 5-year tumor relapse versus those with up to 7-year relapse-free survival, Ascierto et al. ${ }^{52}$ screened more than 299 immune-related genes and found that five genes (IGK [IGKC], GBP1, STAT1, IGLL5, and OCLN) were highly overexpressed in patients with relapse-free survival, highlighting their potential as predictive biomarkers. Similarly, RNA expression studies in ipilimumab-treated patients revealed that the number of immune-related genes involved in both innate and adaptive responses were overexpressed in patients with better clinical activity compared with nonresponsive patients. This suggests the importance of a pre-existing immune-active TME for better clinical response to ipilimumab. PD-L1 and PD-L2 copy number alterations (CNA) are also considered potential biomarkers ${ }^{53}$. Budczies et al. ${ }^{54}$ reported PD-L1 CNA in 22 major cancers and found a strong correlation between PD-L1 CNA and mRNA expression levels. The mutation load also correlated with PD-L1 copy number gains.

The mutational loads in exomes also have potential roles as predictive biomarkers for ICIs. Studies have shown that patients with higher mutational loads have greater responsiveness to ICIs ${ }^{49,55}$. Genetic mutations that lead to the expression of immune-related peptides that expand pre-existing $\mathrm{T}$ cells or that can be generated in response to immune or other stimuli can increase the efficacy of ICIs ${ }^{49,56}$. JAK3, a member of the Janus kinase signaling pathway, generally found in leukocytes, was reported to have a regulatory role in PD-L1 expression in lymphomas ${ }^{57}$. Mutations that activate JAK3 can cause overexpression of PD-L1 in lymphomas and make them responsive to PD-L1 inhibitors ${ }^{58,59}$.

Mismatch-repair mechanisms are the machinery that protects cells by repairing mutations during DNA replications. A high neoantigen load and high mutational load are associated with an improper mismatch-repair 
system. The identification of defective mismatch-repair mechanisms may therefore be exploited as potential predictive biomarkers. Mismatch-repair deficiency in pembrolizumab-treated patients with hereditary nonpolyposis colorectal cancer resulted in a high positive response, highlighting the potential of mismatch-repair deficiency as a predictive biomarker ${ }^{60,61}$. Additionally, in a recent study with 53 cancer patients, the objective response rate was $50 \%$ in patients with mismatch-repair deficiency, compared to $0 \%$ in patients with mismatchproficient tumors ${ }^{60}$. The mismatch-deficient group, compared with the other group, also showed a longer progression-free survival ${ }^{61}$. Advances in NGS and microarray technologies have made genome-wide screening of potential markers comparatively easier. The accurate prediction of these biomarkers and their use in clinical conditions are suboptimal. However, the development of simple algorithms to read these potential gene signatures from patient DNA is necessary to make these findings clinically applicable. A PanCancer IO $360^{\text {tm }}$ assay was developed by nanoString; the assay profiles TME interactions using a 770 gene panel. This panel evaluates multiple immune processes, including simultaneous assessment of immune evasion in the context of all three immune phenotypes (immune desert, immune excluded and immune inflamed) and supports the prediction of patient responses to a variety of immunotherapies, including $\mathrm{ICIs}^{41}$.

\section{Epigenetic signatures}

Epigenetic modifications are complex cellular processes that can modify cellular functions in response to the prevailing environment without altering genetic codes. Multiple epigenetic marks are involved in these complex mechanisms, including DNA methylation, posttranscriptional histone tail modifications, and short noncoding $\mathrm{RNAs}^{62}$. Although the association of multiple epigenetic regulatory mechanisms was evaluated in response to immune checkpoint expression and their applicability in combination therapy for synergistic combination, studies on the evaluation of epigenetic modifications as predictive biomarkers are warranted.

The transcriptomic and epigenetic studies on NSCLC show that the hypomethylation of the CTLA-4, PD-1, and PD-L1 promoter regions may be associated with the upregulation of these genes in the $\mathrm{TME}^{63}$. It has been shown that in chronic lymphocytic leukemia (CLL), the mRNA and protein levels of PD-1 were elevated and significantly hypomethylated in both promoter and enhancer regions compared to healthy B-cell controls ${ }^{64}$.

miRNAs are small single-stranded RNA sequences that have a critical role in various diseases, including cancer ${ }^{65}$. Reports have shown that five members of the miR-200 family, miR-200a, 200b, 200c, 141, and 429, play pivotal roles in tumor suppression by restricting the epithelial-tomesenchymal transition (EMT) ${ }^{66-68}$. In human breast cancer cells, it has been reported that expression of PD-L1 decreases with overexpression of miR-200 ${ }^{69}$. These reports rationalize the hypothesis that miR-200 might be a promising biomarker for responders treated with anti-PDL1 antibodies (atezolizumab or durvalumab). A recent study showed that serum miRNA levels correlated with progression-free survival and overall survival in a phase II clinical study on patients with esophageal squamous cell carcinoma (ESCC) treated with nivolumab ${ }^{70}$. Eight miRNAs were found to be associated with a better clinical response, out of which four miRNAs were positively associated with progression-free survival ${ }^{70}$. In contrast, overexpression of miR-34a has been reported as an inducer of $\mathrm{CD}^{+}$TILs by repressing PD-L1 expression in colorectal carcinoma and NSCLC patients ${ }^{71,72}$. These data suggest that the miRNA-PD-L1 axis might be a promising therapeutic/diagnostic biomarker target in ICI therapy.

\section{Concluding remarks}

Immunological response to ICIs is a complex process. Biomarkers that predict the efficacy of ICI therapy and irAEs should help in patient selection and decisionmaking by distinguishing between responders and nonresponders. Numerous studies on predictive biomarkers focusing on immune cell infiltration, peripheral blood analyses, PD-L1 overexpression, copy number alterations, neoantigen clonality, mutational landscape, mismatchrepair deficiency, SNPs, transcription factors, and miRNA are currently available (Table 2).

Major issues in the development of predictive biomarkers are the dynamic variations in cancer biomarker types and a patient's genetic makeup. Biopsies obtained from multiple sites of the same patient showed variation in biomarker levels owing to intratumoral heterogeneity. Intense research will develop combination biomarker sets to predict ICI therapy outcomes and avoid irAEs ${ }^{73}$.

Although several predictive biomarker studies are completed and many are underway, the clinical validation of the identified biomarkers is necessary. More integrated approaches should be developed to identify patientspecific choices for checkpoint monotherapies or combination therapies. Moreover, next-generation sequencing techniques should become clinically applicable through the development of simple algorithms to process large quantities of clinical data. In conclusion, biomarkerdriven prediction of immune therapy outcomes has the potential to make dramatic changes in cancer immunotherapy.

\section{Acknowledgements}

This work was supported by a start-up grant [VR04] for Dr. Eyad Elkord from Qatar Biomedical Research Institute, Qatar Foundation. 
Table 2 Predictive biomarkers for progression-free survival and overall survival in patients treated with immune checkpoint inhibitors

\begin{tabular}{|c|c|c|}
\hline Biomarker Category & Nonresponders & Responders \\
\hline Immune cells & $\begin{array}{l}\text { Decreased } \\
\text { - Lymphocytes }\left(\mathrm{CD}^{+}, \mathrm{CD} 8^{+}\right)^{14} \\
\text { - } \text { B Cells }\left(\mathrm{CD} 20^{+}\right)^{26} \\
\text { - Activated lymphocytes }\left(\mathrm{CD} 134^{+}, \mathrm{CD} 137^{+} \text {, and }\right. \\
\left.\text { FOXP3 }^{+}\right)^{26} \\
\text { - Natural killer cells }\left(\mathrm{NKp} 46^{+}\right)^{26}\end{array}$ & 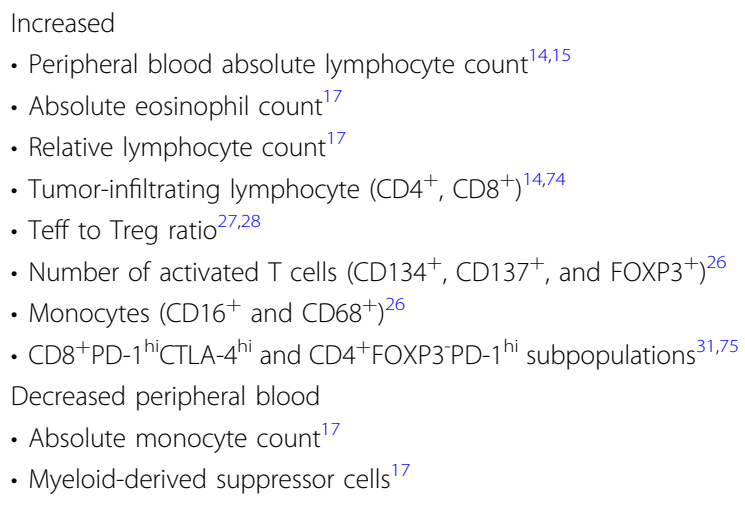 \\
\hline Protein expression & $\begin{array}{l}\text { - Basal level expression of PD-L } 1^{43} \\
\text { - The loss in IFN- } \gamma \text { signaling in } \mathrm{CD}^{+} \mathrm{T} \text { cells }{ }^{47}\end{array}$ & 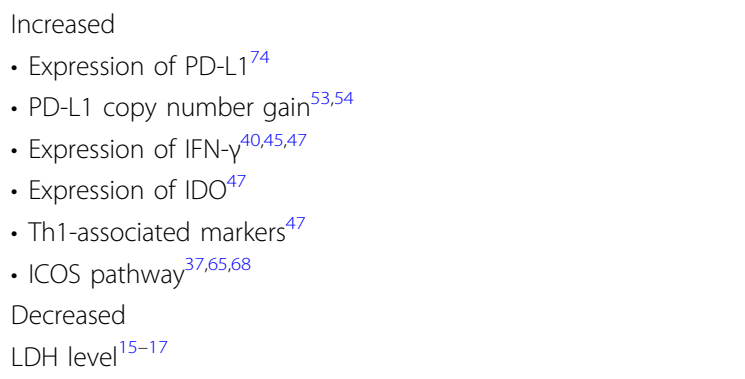 \\
\hline Mutations and neoantigens & $\begin{array}{l}\text { - Elimination of neoantigen-expressing tumor } \\
\text { clones }^{48} \\
\text { - Decreased neoantigen burden }{ }^{47,76}\end{array}$ & $\begin{array}{l}\text { - Higher mutational load }{ }^{49,55} \\
\text { - Clonal mutations in neoantigens }{ }^{47,48} \\
\text { - Mismatch-repair deficiency }{ }^{60,61} \\
\text { - Increased neoantigen burden }{ }^{47}\end{array}$ \\
\hline Gene signatures & & $\begin{array}{l}\text { - Overexpression of IGK, GBP1, STAT1, IGLL5, and OCLN }{ }^{52} \\
\text { - Overexpression of immune-related peptides expanding pre- } \\
\text { existing T cells }{ }^{49,56} \\
\text { - Activation in JAK3 }{ }^{58,59}\end{array}$ \\
\hline Epigenetic signatures & & $\begin{array}{l}\text { - Altered methylation pattern of PD-L1 } 26,63,64 \\
\text { - Higher serum levels of miRNA }\end{array}$ \\
\hline
\end{tabular}

\section{Conflict of interest}

The authors declare that they have no conflict of interest.

\section{Publisher's note}

Springer Nature remains neutral with regard to jurisdictional claims in published maps and institutional affiliations.

Received: 9 June 2018 Revised: 22 August 2018 Accepted: 19 September 2018.

Published online: 13 December 2018

\section{References}

1. Hodi, F. S. et al. Improved survival with ipilimumab in patients with metastatic melanoma. N. Engl. J. Med. 363, 711-723 (2010).

2. Robert, $C$. et al. Ipilimumab plus dacarbazine for previously untreated metastatic melanoma. N. Engl. J. Med. 364, 2517-2526 (2011).
3. Gibney, G. T., Weiner, L. M. \& Atkins, M. B. Predictive biomarkers for checkpoint inhibitor-based immunotherapy. Lancet Oncol. 17, e542-e551 (2016).

4. Borghaei, $\mathrm{H}$. et al. Nivolumab versus docetaxel in advanced nonsquamous non-small-cell lung cancer. N. Engl. J. Med. 373, 1627-1639 (2015).

5. Garon, E. B. et al. Pembrolizumab for the treatment of non-small-cell lung cancer. N. Engl. J. Med. 372, 2018-2028 (2015).

6. Larkin, J. et al. Combined nivolumab and ipilimumab or monotherapy in untreated melanoma. N. Engl. J. Med. 373, 23-34 (2015).

7. Cheng, W., Fu, D., Xu, F. \& Zhang, Z. Unwrapping the genomic characteristics of urothelial bladder cancer and successes with immune checkpoint blockade therapy. Oncogenesis 7, 2 (2018).

8. Polk, A., Svane, I. -M., Andersson, M. \& Nielsen, D. Checkpoint inhibitors in breast cancer: Current status. Cancer Treat. Rev. 63, 122-134 (2013).

9. Ansell, S. M. et al. PD-1 Blockade with nivolumab in relapsed or refractory Hodgkin's lymphoma. N. Engl. J. Med. 372, 311-319 (2014).

10. Chen, D. S. \& Mellman, I. Elements of cancer immunity and the cancer-immune set point. Nature 541, 321 (2017).

11. Feng, $Y$. et al. Exposure-response relationships of the efficacy and safety of ipilimumab in patients with advanced melanoma. Clin. Can. Res. 19, 3977 (2013). 
12. Cogdill, A. P., Andrews, M. C. \& Wargo, J. A. Hallmarks of response to immune checkpoint blockade. Br. J. Cancer 117, 1-7 (2017).

13. Pagès, F. et al. Effector memory T cells, early metastasis, and survival in colorectal cancer. N. Engl. J. Med. 353, 2654-2666 (2005).

14. Angulo, G. D., Yuen, C., Palla, S. L., Anderson, P. M. \& Zweidler-McKay, P. A. Absolute lymphocyte count is a novel prognostic indicator in ALL and AML. Cancer 112, 407-415 (2008).

15. Simeone, E. et al. Immunological and biological changes during ipilimumab treatment and their potential correlation with clinical response and survival in patients with advanced melanoma. Cancer Immunol. Immunother. 63 675-683 (2014)

16. Kelderman, S. et al. Lactate dehydrogenase as a selection criterion for ipilimumab treatment in metastatic melanoma. Cancer Immunol. Immunother. $\mathbf{6 3}$ 449-458 (2014).

17. Martens, A. et al. Baseline peripheral blood biomarkers associated with clinical outcome of advanced melanoma patients treated with ipilimumab. Clin. Can. Res. 22, 2908 (2016).

18. Buder-Bakhaya, K. \& Hassel, J. C. Biomarkers for clinical benefit of immune checkpoint inhibitor treatment-a review from the melanoma perspective and beyond. Front. Immunol. 9, 1474 (2018).

19. Manola, J., Atkins, M., Ibrahim, J. \& Kirkwood, J. Prognostic factors in metastatic melanoma: A pooled analysis of eastern cooperative oncology group trials. J. Clin. Oncol. 18, 3782-3793 (2000).

20. Subrahmanyam, P. B. et al. Distinct predictive biomarker candidates for response to anti-CTLA-4 and anti-PD-1 immunotherapy in melanoma patients. J. Immunother. Cancer 6, 18 (2018).

21. Krieg, C. et al. High-dimensional single-cell analysis predicts response to antiPD-1 immunotherapy. Nat. Med. 24, 144 (2018).

22. Martens, A. et al. Increases in absolute lymphocytes and circulating CD4(+) and $\mathrm{CD} 8(+) \mathrm{T}$ cells are associated with positive clinical outcome of melanoma patients treated with ipilimumab. Clin. Can. Res 22, 4848-4858 (2016).

23. Wistuba-Hamprecht, K. et al. Peripheral CD8 effector memory type 1 T-cells correlate with outcome in ipilimumab-treated stage $\mathrm{N}$ melanoma patients. Eur. J. Cancer 73, 61-70 (2017).

24. de Coaña, Y. P. et al. Ipilimumab treatment decreases monocytic MDSCs and increases CD8 effector memory T cells in long-term survivors with advanced melanoma. Oncotarget 8, 21539-21553 (2017).

25. Hamid, O. et al. A prospective phase II trial exploring the association between tumor microenvironment biomarkers and clinical activity of ipilimumab in advanced melanoma. J. Transl. Med. 9, 204-204 (2011).

26. Balatoni, T. et al. Tumor-infiltrating immune cells as potential biomarkers predicting response to treatment and survival in patients with metastatic melanoma receiving ipilimumab therapy. Cancer Immunol. Immunother. 67, 141-151 (2018).

27. Quezada, S. A., Peggs, K. S., Curran, M. A. \& Allison, J. P. CTLA4 blockade and GM-CSF combination immunotherapy alters the intratumor balance of effector and regulatory T cells. J. Clin. Invest. 116, 1935-1945 (2006).

28. Hodi, F. S. et al. Immunologic and clinical effects of antibody blockade of cytotoxic T lymphocyte-associated antigen 4 in previously vaccinated cancer patients. Proc. Natl Acad. Sci. 105, 3005-3010 (2008).

29. Daud, A. I. et al. Tumor immune profiling predicts response to anti-PD-1 therapy in human melanoma. J. Clin. Invest. 126, 3447-3452 (2016).

30. Thommen, D. S. et al. A transcriptionally and functionally distinct PD-1 + CD8 $+T$ cell pool with predictive potential in non-small-cell lung cancer treated with PD-1 blockade. Nat. Med. 24, 994-1004 (2018).

31. Zappasodi, R. et al. Non-conventional inhibitory CD4 $4^{+}$Foxp3 ${ }^{-}$PD- $1^{\text {hi }} T$ cells as a biomarker of immune checkpoint blockade activity. Cancer Cell 33, 1017-1032.e1017 (2018).

32. Wu, S. P. et al. Stromal PD-L1 positive regulatory T cells and PD-1 positive CD8positive $T$ cells define the response of different subsets of non-small cell lung cancer to PD-1/PD-L1 blockade immunotherapy. J. Thorac. Oncol. 13, 521-532 (2018).

33. Tumeh, P. C. et al. PD-1 blockade induces responses by inhibiting adaptive immune resistance. Nature 515, 568 (2014).

34. Geng, Y. et al. Prognostic role of tumor-infiltrating lymphocytes in lung cancer: A meta-analysis. Cell. Physiol. Biochem. 37, 1560-1571 (2015).

35. Schmid, P. et al. Atezolizumab in metastatic TNBC (mTNBC): Long-term clinical outcomes and biomarker analyses. Cancer Res. 77, 2986 (2017). abstr.

36. Loi, S. et al. LBA13 relationship between tumor infiltrating lymphocyte (TIL) levels and response to pembrolizumab (pembro) in metastatic triple-negative breast cancer (mTNBC): Results from KEYNOTE-086. Ann. Oncol. 28, $m d x 440.005-m d x 440.005$ (2017)

37. Liakou, C. I. et al. CTLA-4 blockade increases IFNy-producing CD4(+)ICOS(hi) cells to shift the ratio of effector to regulatory $T$ cells in cancer patients. Proc. Natl Acad. Sci. 105, 14987-14992 (2008).

38. Tang, D. N. et al. Increased frequency of ICOS + + ) CD4 T-cells as a pharmacodynamic biomarker for anti-CTLA-4 therapy. Cancer Immunol. Res. 1, 229-234 (2013).

39. Chen, $\mathrm{H}$. et al. Anti-CTLA-4 therapy results in higher $\mathrm{CD}^{+}{ }^{\mathrm{I}} \mathrm{COS}{ }^{\mathrm{hi}} \mathrm{T}$ cell frequency and IFN- $\gamma$ levels in both nonmalignant and malignant prostate tissues. Proc. Natl Acad. Sci. 106, 2729 (2009).

40. Chen, $\mathrm{H}$. et al. CD4 T cells require ICOS-mediated PI3K-signaling to increase Tbet expression in the setting of anti-CTLA-4 therapy. Cancer Immunol. Res. 2, 167-176 (2014).

41. Cesano, A. \& Warren, S. Bringing the next generation of immuno-oncology biomarkers to the clinic. Biomedicines 6, 14 (2018).

42. Toor, S. M. \& Elkord, E. Therapeutic prospects of targeting myeloid-derived suppressor cells and immune checkpoints in cancer. Immunol. Cell Biol. 96, 888-897 (2018).

43. Teng, M. W., Ngiow, S. F., Ribas, A. \& Smyth, M. J. Classifying cancers based on T-cell infiltration and PD-L1. Cancer Res. 75, 2139-2145 (2015).

44. Reck, M. et al. Pembrolizumab versus chemotherapy for PD-L1-positive non-small-cell lung cancer. N. Engl. J. Med. 375, 1823-1833 (2016).

45. Maleki Vareki, S., Garrigos, C. \& Duran, I. Biomarkers of response to PD-1/PD-L1 inhibition. Crit. Rev. Oncol. Hematol. 116, 116-124 (2017).

46. Guibert, N. et al. PD-L1 expression in circulating tumor cells of advanced nonsmall cell lung cancer patients treated with nivolumab. Lung Cancer 120, 108-112 (2018).

47. McGranahan, N. et al. Clonal neoantigens elicit T cell immunoreactivity and sensitivity to immune checkpoint blockade. Science 351, 1463-1469 (2016).

48. Anagnostou, V. et al. Evolution of neoantigen landscape during immune checkpoint blockade in non-small cell lung cancer. Cancer Discov. 7, 264-276 (2017).

49. Snyder, A. et al. Genetic basis for clinical response to CTLA-4 blockade in melanoma. N. Engl. J. Med. 371, 2189-2199 (2014).

50. Jenkins, R. W., Barbie, D. A. \& Flaherty, K. T. Mechanisms of resistance to immune checkpoint inhibitors. Br. J. Cancer 118, 9-16 (2018).

51. Zaretsky, J. M. et al. Mutations associated with acquired resistance to PD-1 blockade in melanoma. N. Engl. J. Med. 375, 819-829 (2016).

52. Ascierto, M. L. et al. A signature of immune function genes associated with recurrence-free survival in breast cancer patients. Breast Cancer Res. Treat. 131 $871-880$ (2012)

53. Inoue, Y. et al. Clinical significance of PD-L1 and PD-L2 copy number gains in non-small-cell lung cancer. Oncotarget 7, 32113-32128 (2016).

54. Budczies, J. et al. Pan-cancer analysis of copy number changes in programmed death-ligand 1 (PD-L1, CD274) - associations with gene expression, mutational load, and survival. Genes Chromosomes Cancer 55, 626-639 (2016).

55. Rizvi, N. A. et al. Mutational landscape determines sensitivity to PD-1 blockade in non-small cell lung cancer. Science 348, 124 (2015).

56. Gnjatic, S. et al. Identifying baseline immune-related biomarkers to predict clinical outcome of immunotherapy. J. Immunother. Cancer 5, 44 (2017).

57. Green, M. R. et al. Constitutive AP-1 activity and EBV infection induce PD-L1 in Hodgkin lymphomas and posttransplant lymphoproliferative disorders: Implications for targeted therapy. Clin. Can. Res. 18, 1611 (2012).

58. Topalian, S. L., Taube, J. M., Anders, R. A. \& Pardoll, D. M. Mechanism-driven biomarkers to guide immune checkpoint blockade in cancer therapy. Nat. Rev. Cancer 16, 275 (2016).

59. Van Allen, E. M. et al. Long-term benefit of PD-L1 blockade in lung cancer associated with JAK3 activation. Cancer Immunol. Res. 3, 855 (2015).

60. Le, D. T. et al. PD-1 blockade in tumors with mismatch-repair deficiency. $N$. Engl. J. Med. 372, 2509-2520 (2015).

61. Le, D. T. et al. Mismatch-repair deficiency predicts response of solid tumors to PD-1 blockade. Science 357, 409-413 (2017).

62. Wright, J. Epigenetics: reversible tags. Nature 498, S10-11 (2013).

63. Marwitz, S. et al. Epigenetic modifications of the immune-checkpoint genes CTLA4 and PDCD1 in non-small cell lung cancer results in increased expression. Clin. Epigenetics 9, 51 (2017).

64. Xu-Monette, Z. Y., Zhou, J. \& Young, K. H. PD-1 expression and clinical PD-1 blockade in B-cell lymphomas. Blood 131, 68-83 (2018).

65. Croce, C. M. Causes and consequences of microRNA dysregulation in cancer. Nat. Rev. Genet. 10, 704-714 (2009). 
66. Mongroo, P. S. \& Rustgi, A. K. The role of the miR-200 family in epithelialmesenchymal transition. Cancer Biol. Ther. 10, 219-222 (2010).

67. Korpal, M., Lee, E. S., Hu, G. \& Kang, Y. The miR-200 family inhibits epithelialmesenchymal transition and cancer cell migration by direct targeting of $\mathrm{E}$ cadherin transcriptional repressors ZEB1 and ZEB2. J. Biol. Chem. 283, 14910-14914 (2008)

68. Huber, M. A. et al. NF-kappaB is essential for epithelial-mesenchymal transition and metastasis in a model of breast cancer progression. J. Clin. Invest. 114, 569-581 (2004)

69. Noman, M. Z. et al. The immune checkpoint ligand PD-L1 is upregulated in EMT-activated human breast cancer cells by a mechanism involving ZEB-1 and miR-200. Oncoimmunology 6, e1263412 (2017).

70. Sudo, K. et al. Serum microRNAs to predict the response to nivolumab in patients with esophageal squamous cell carcinoma. J. Clin. Oncol. 35, e14511-e14511 (2017).
71. Li, X., Nie, J., Mei, Q. \& Han, W. D. MicroRNAs: novel immunotherapeutic targets in colorectal carcinoma. World J. Gastroenterol. 22, 5317-5331 (2016).

72. Cortez, M. A. et al. PD-L1 regulation by p53 via miR-34. J. Natl. Cancer Inst. 108 djv303 (2016).

73. Postow, M. A., Sidlow, R. \& Hellmann, M. D. Immune-related adverse events associated with immune checkpoint blockade. N. Engl. J. Med. 378, 158-168 (2018).

74. Patel, S. P. \& Kurzrock, R. PD-L1 expression as a predictive biomarker in cancer immunotherapy. Mol. Cancer Ther. 14, 847 (2015).

75. Galon, J. et al. Type, density, and location of immune cells within human colorectal tumors predict clinical outcome. Science 313, 1960 (2006).

76. Aguiar, P. N. et al. The role of PD-L1 expression as a predictive biomarker in advanced non-small-cell lung cancer: a network meta-analysis. Immunotherapy 8, 479-488 (2016) 